\title{
Les débuts de la fonte ornementale en France, de l'Empire à la monarchie de Juillet
}

The Early Days of Ornamental Cast Iron in France, from the Empire to the July Monarchy

Jean-Paul Zitt

\section{(2) OpenEdition} Journals

Édition électronique

URL : http://journals.openedition.org/cel/652

DOI : $10.4000 /$ cel. 652

ISSN : 2262-208X

Éditeur

École du Louvre

Édition imprimée

Date de publication : 1 septembre 2012

Référence électronique

Jean-Paul Zitt, « Les débuts de la fonte ornementale en France, de l'Empire à la monarchie de Juillet », Les Cahiers de l'École du Louvre [En ligne], 1 | 2012, mis en ligne le 01 septembre 2012, consulté le 01 mai 2019. URL : http://journals.openedition.org/cel/652; DOI : 10.4000/cel.652

\section{c) (i) (2)}

Les Cahiers de l'École du Louvre sont mis à disposition selon les termes de la licence Creative Commons Attribution - Pas d'Utilisation Commerciale - Pas de Modification 4.0 International. 


\title{
Les débuts de la fonte ornementale en France, de l'Empire à la monarchie de Juillet
}

\author{
Jean-Paul Zitt
}

\begin{abstract}
«César, sa femme et Césarine sortirent de la boutique et entrèrent chez eux par la rue. La porte de la maison avait été refaite dans un grand style, à deux vantaux divisés par des panneaux égaux et carrés, au milieu desquels se trouvait un ornement architectural de fonte coulée et peinte. Cette porte, devenue si commune à Paris, était alors dans toute sa nouveauté. »
\end{abstract}

Balzac, César Birotteau ${ }^{1}$

Si la fonte de fer est connue depuis le Moyen Âge, son essor coïncide avec la révolution industrielle et la modernisation de la ville sous l'Empire et la Restauration. Matériau résistant adapté à la construction, il sait aussi par sa technique même de fabrication, le moulage, se plier à une infinie variété de formes. Cette double nature lui permet d'investir le décor des immeubles bourgeois, des théâtres et des galeries ouvertes à un public féru de salubrité, de confort et d'agrément. Sa qualité en progrès rapide, sa malléabilité, inspirent architectes et sculpteurs. La fonte ornementale est-elle un art? Des contemporains lui reprochent son manque de sincérité2 $\ldots$

\section{À la recherche de la fonte malléable}

Jusqu'au XVIe siècle, l'alliage liquide qui s'écoule occasionnellement du fourneau, durcissant rapidement, cassant, est considéré comme inapte à toute utilisation économique. Les anciens ornements en fonte de fer des portes de Notre-Dame de Paris étaient perçus comme si extraordinaires qu'on eût cru que le diable s'en était mêlé3. On parle de «secret perdu». L'obtention d'une fonte résistante, épousant bien le moule et se prêtant à une finition ultérieure, reste hors de portée. Au XVI e siècle, avec la révolution du haut-fourneau ${ }^{4}$, le matériau entre en concurrence avec le fer, le bronze et la terre cuite pour des applications utilitaires, comportant parfois un décor en faible relief : contrecœurs de cheminée, canons, vases... L'édition de 1710 du Cours d'architecture de d'Aviler illustre pour la première fois un balcon de fer fondu, ainsi que la luxueuse rampe de l'hôtel Le Peletier de Saint-Fargeau toujours visible in situ. Au même moment, malgré les limites afférentes aux connaissances du temps ${ }^{5}$, Réaumur progresse dans l'obtention d'une fonte "adoucie ${ }^{6}$. Il met en pratique ses découvertes à la Manufacture royale d'Orléans, qui propose vers 1725 dans son magasin parisien un assortiment de balcons, rampes, vases et marteaux de porte aux décors parfois ornés de figures en ronde-bosse ${ }^{7}$.

\footnotetext{
1 Édition GF-Flammarion, Paris, 1995, p. 192.

${ }^{2}$ Cet article reprend des thèmes développés dans le mémoire de seconde année de $2^{\mathrm{e}}$ cycle de l'École du Louvre: Jean-Paul Zitt, «Les débuts de la fonte ornementale en France, du Premier Empire à la monarchie de Juillet », septembre 2011.

3 Jacques-Antoine Dulaure, Histoire civile, physique et morale de Paris, v. 2, Paris, Baudouin frères, 1825 , p. 340.

4 Consulter notamment Jean-François Belhoste (coll.), "La métallurgie normande, XII XVII ${ }^{e}$ siècles : la révolution du haut-fourneau ", Cahiers de l'inventaire, $\mathrm{n}^{\circ} 14,1991$.

${ }^{5}$ Notamment l'incompréhension d'un paramètre majeur : le taux de carbone.

${ }^{6}$ Pouvant être limée ou percée après moulage.

7 État des Ouvrages de Fer \& Acier fondus \& adoucis..., Paris, de I'imprimerie de d'Houry, 1727 (conservé à la bibliothèque Mazarine, $4^{\circ} 10371 \mathrm{P}$ pièce 80 ); voir Daniel Bontemps et Catherine Prade : “Un magasin parisien d'ouvrages en fonte de fer ornée au XVIII ${ }^{\ominus}$ siècle, une réussite métallurgique méconnue de Réaumur ", Bulletin de la Société d'histoire de Paris et de I'T̂le-de-France, $118^{\mathrm{e}}$ année, 1991, Paris, École nationale des Chartes, 1994, pp. 215-261.
} 
L'opposition de la corporation des serruriers et diverses difficultés mettent un terme à l'expérience. Sous l'Empire, les problèmes rencontrés par l'architecte Vaudoyer pour la fonte des lionnes de la fontaine de l'Institut (fig. 1) ${ }^{8}$ montrent que le matériau, s'il commence à s'affirmer dans la construction, est difficile à maitriser en sculpture monumentale. C'est finalement la fonderie du Creusot, alors à la pointe de la technique, qui livre les quatre statues9. Dans la décennie 1820, la fabrication de la fonte va rapidement atteindre la maturité, stimulée par l'amélioration de la production des hauts-fourneaux, la maitrise progressive des processus de deuxième fusion et de moulage ${ }^{10}$, et la demande croissante.

\section{Une double nature}

Ces obstacles levés, la fonte se révèle indispensable. Quel autre matériau peut répondre à coût économique à la variété des besoins ? Un commerçant ouvre boutique dans une des galeries nouvelles: offrant la résistance de massifs piliers maçonnés, deux colonnettes en fonte permettent d'élargir la devanture pour mettre en valeur l'assortiment. Les structures métalliques qui apparaissent alors dans la ville frappent les contemporains qui y voient soit une architecture de la légèreté, soit la laideur de lignes grêles s'opposant aux volumes harmonieux des monuments de pierre. Ponts, fontaines, colonnes, escaliers font l'objet d'une ornementation quasi systématique ; la technique s'y prête, produisant en une même opération structure et ornement. La flèche de la cathédrale de Rouen, élevée à partir de 1827 sur les plans de l'architecte Jean-Antoine Alavoine, est un défi sans précédent. La résistance mécanique de la fonte permet de concevoir un ouvrage de 148 mètres, prolongeant l'édifice

8 Elles se trouvent aujourd'hui dans un square de Boulogne-Billancourt; voir Dominique Perchet, "Aux origines de la fonte d'art", Fontes n 73 (revue de l'Association pour la sauvegarde du patrimoine métallurgique haut-marnais), p. 13.

${ }^{9}$ Paris, Archives nationales, $\mathrm{F}^{13} 1002$.

${ }^{10}$ Deux mémoires du fondeur Calla, "Note sur un moyen de rendre la fonte malléable " et "Mémoire sur la fabrication des fers fondus", publiés en 1827 et 1831 dans le Bulletin de la Société d'Encouragement, témoignent du passage de méthodes empiriques à une approche rationnelle en phase avec la révolution industrielle. 
gothique dans le jeu des pleins et des vides et la verticalité ${ }^{11}$. Dix ans plus tard, au Jardin des Plantes, les serres de l'architecte Charles Rohault de Fleury sont emblématiques des capacités de la fonte à s'insérer dans une architecture nouvelle associant élégance et fonctionnalité. La ville a besoin d'eau: les fontaines sont un terrain de prédilection pour le matériau, aussi présent dans la partie visible, vasques et figures, que dans la partie invisible, pour le réseau d'alimentation. Les meilleurs fondeurs parviennent à lui donner une netteté12 comparable à celle du bronze. L'exigence de sécurité contre l'incendie en favorise l'emploi pour les colonnes, balustrades et escaliers, qui se prêtent à des variations décoratives. Les élégants escaliers de fonte de la galerie du PalaisRoyal de 1828 permettent à travers leur noyau creux l'évacuation des fumées produites par les calorifères (fig. 2). Le lieu bénéficie d'une autre innovation: « cet inappréciable éclairage au gaz, dans lequel les candélabres en fonte jouent un rôle aussi nécessaire que pittoresque $»^{13}$. Fontaine commente les travaux en cours : «Nous espérons qu'avec un produit assuré d'au moins 200 mille francs et l'affluence continuelle des curieux que cet édifice attire, on nous saura quelque gré de nos efforts ${ }^{14}$. La fonte ornementale participe pleinement à cette logique de rentabilité.

11 «La ville de Rouen aurait la gloire de posséder un édifice dépassant [la pyramide de Khéops] de 2 mètres 629 millimètres, et qui, ainsi, deviendrait le plus haut du monde entier ", E. de la Quérière, La Flèche en fer fondu de la cathédrale de Rouen, Rouen, Imprimerie de D. Brière, 1856 , p. 2. Voir aussi Jean-Philippe Desportes, "Alavoine et la flèche de la cathédrale de Rouen", Revue de l'Art, $\mathrm{n}^{\circ}$ 13, 1971, pp. 46-63. La flèche ne serait achevée qu'en 1876.

${ }^{12}$ Les fontes de Calla sont primées à l'Exposition des produits de l'industrie française de 1839 pour "la netteté de leur exécution au moulage".

${ }^{13}$ Charles Louis Gustave Eck. Traité de l'application du fer, de la fonte et de la tôle dans les constructions civiles, industrielles et militaires..., Paris, Carilian-Goeury, 1841, p. 76.

${ }_{14}$ Pierre François Léonard Fontaine, Journal, 1799-1853, Paris, École nationale supérieure des Beaux-Arts, Institut français d'Architecture, Société de I'Histoire de I'art français, 1987, p. 788 (journée du 8 décembre 1828). 


\section{Matières, formes et couleurs nouvelles dans la ville}

Construit en 1803, le pont des Arts affirme face au Pont-Neuf des lignes radicalement nouvelles. Vingt ans plus tard, le Journal des artistes commente ainsi la dernière réalisation de l'ingénieur Polonceau : «Sous le rapport monumental, le pont du Carrousel est remarquable aussi ; l'extrême légèreté de ses voûtes à jour, de sa balustrade grillée, l'immense ouverture de ses arches, lui donnent un style tout spécial» ${ }^{15}$. L'élégance du dessin et la modernité de l'ouvrage de métal lui méritent sa place devant le Louvre. Non loin de là, galerie d'Orléans, le visiteur entre dans un univers inédit : sols de marbre, toiture en fer et verre, éclairage au gaz, boutiques luxueuses... Fonctionnel, salubre, accessible à tous, le lieu est un manifeste de la ville nouvelle ${ }^{16}$. La fonte s'intègre naturellement au système voulu par Fontaine, et si l'architecte passe pour ne pas aimer le métal, il lui fait ici la part belle : combles, escaliers, candélabres, balustres, caisses à orangers... Jouant des contrastes de matières et de couleurs, le décor des théâtres, des passages et des boutiques évolue au gré de la fantaisie des propriétaires. La fonte s'y épanouit, elle est à la mode: "Quant à l'effet comme ornement, la fonte peinte et bronzée est bien supérieure au bois et à la pierre $»^{17}$. Le nouveau bazar Montesquieu « se compose de 80 comptoirs d'une forme élégante et séparés par des colonnes de fonte, recouvertes en cuivre cannelé et surmontées d'un ornement en cuivre estampé. Tous ces objets, d'un travail et d'un poli remarquable, offrent à l'œil un ensemble d'une délicatesse et d'un goût exquis ${ }^{18}$. » Avec les fontaines des Mers et des Fleuves de la place de la Concorde ${ }^{19}$ (fig. 3), le matériau semble approcher de ses limites techniques ${ }^{20}$, mais prouve qu'il peut rivaliser avec la pierre ou le bronze des places royales de l’Ancien Régime.

\footnotetext{
15 Journal des artistes, 1834, v. 2, n¹9, p. 291.

16 "Les passages Vérot-Dodat, Vivienne, Colbert, la galerie d'Orléans, et ses jolies boutiques, avec leurs gracieux et hardis escaliers en fonte [...] ce sont là, sans doute, des utiles et attirantes expositions des produits de l'industrie ", Stéphane Flachat, L'Exposition des produits de l'industrie en 1834, Paris, L. Tenré, 1834, p. 16.

17 Journal des connaissances usuelles et pratiques, t. 17, Paris, MM. de Grandmont et de Lasteyrie, 1833, p. 276.

${ }^{18}$ Bulletin universel des sciences et de l'industrie, bulletin des sciences technologiques, v. 14, Paris, Firm in Didot, 1830 , p. 370.

${ }^{19}$ Inaugurée le $1^{\text {er }}$ mai 1840 .

${ }^{20}$ Comme la lecture du Journal de l'architecte Hittorff le laisse supposer (v. 9, fac-similé consultable à la documentation du musée Carnavalet, Paris).
} 
Au même moment, à l'église Saint-Vincent-de-Paul, l'architecte Jacques-Ignace Hittorff met en scène matières, formes et couleurs dans une architecture polychrome inspirée de l'Antiquité. Portes monumentales, grilles, bénitiers, torchères, candélabres et frises du fondeur Calla y voisinent avec les laves émaillées de Jollivet et les peintures murales de Flandrin dans une harmonie nouvelle (fig. 4).

\section{Fondeurs et catalogues}

Les fondeurs de fer sont peu nombreux à Paris, moins d'une dizaine sous l'Empire, une trentaine au plus sous la monarchie de Juillet ${ }^{21}$. Si le développement de la deuxième fusion permet de s'affranchir des hautsfourneaux, elle reste affaire de spécialiste. La décennie 1820 voit un changement radical de la demande: il faut meubler la ville, décorer les nouveaux immeubles, orner en série. La production se rationalise, les produits se standardisent. Des acteurs nouveaux apparaissent, d'autres évoluent ou disparaissent. André, Ducel père et fils ${ }^{22}$, Calla père et fils, Latron, Savanne et quelques autres, capables de mobiliser les moyens humains, techniques et financiers nécessaires, font en quelques années basculer le métier de l'artisanat à l'industrie ${ }^{23}$. Proches des architectes et des commanditaires, ils côtoient sculpteurs et ornemanistes sur les mêmes chantiers. À la fois industriels et artistes, François-Etienne et Christophe-François Calla interviennent aux Tuileries, au Palais-Royal, au Cirque d'Été, au bazar Montesquieu, au Panthéon, à Notre-Dame-de-Lorette et Saint-Vincent-de-Paul ${ }^{24} \ldots$

La Manufacture d'Orléans proposait déjà au public une liste tarifée de ses produits, mais il s'agissait, sinon de pièces uniques, du moins de très petites

${ }^{21}$ Voir I'Almanach du Commerce.

${ }^{22}$ Qui proposent les premiers, dès 1825, des fontes ornementales dans leur annonce de I'Almanach.

${ }^{23}$ Pour plus d'informations sur les fondeurs, se référer au mémoire « Les débuts de la fonte ornementale en France » (op. cit.), chapitres «Les fondeurs de fer au début du XIXe siècle ", pp. 28-30, et "Éléments sur quelques fondeurs actifs sur le marché parisien », pp. 81-95 ; consulter également le site internet du Réseau international de la fonte d'art: www. fontesdart.org.

${ }^{24}$ Voir notamment Jules Gaudry: «Notice nécrologique sur Christophe-François Calla ", Mémoires et compte rendu des travaux de la Société des ingénieurs civils, premier volume, année 1884 , p. 370. 
séries. C’est vers 1820 que la nécessité pratique de planches illustrées et cotées a dû se faire jour : l'ornement en série est sans doute à l'origine du catalogage. Trois catalogues anciens ont été retrouvés, des fondeurs Jean-Pierre Victor André (fig. 5), Michel Savanne et Jean-Jacques Ducel25. Le type de mesures utilisé, les lithographes ainsi que l'adresse des établissements suggèrent des assemblages vers 1838-1840, certaines planches pouvant remonter à la fin de la décennie antérieure. Organisés par famille, illustrés, tarifés, ils ne sont pas très différents des catalogues d'aujourd'hui. Les dimensions standardisées permettent des assemblages variés à partir de modules et d'éléments de base, facilitant les choix du client, de l'architecte ou du maçon. Leur présentation n'est pas homogène : y cohabitent mesures anciennes et métriques, caractères typographiques et écriture manuelle. Le temps et le coût nécessaire à la gravure ou à la lithographie ont dû faire de l'édition de chaque planche une décision importante, et l'actualisation se fait par ajout plutôt que par refonte. La richesse du catalogue de Ducel montre les progrès rapides de la nouvelle industrie ; elle pose aussi la question des modèles. 


\section{Modèles, cahiers d'ornements et traités d'architecture}

Si les ouvrages de prestige sont le fait d'architectes et d'artistes reconnus comme Fontaine, Hittorff, Visconti, Pradier, Klagmann ou Bosio, quels sont les modèles des panneaux, chapiteaux, frises, mascarons et fontaines des catalogues ? Le néo-classicisme des trophées s'y mêle aux arabesques, aux oiseaux exotiques d'inspiration persane, aux têtes pittoresques de la Renaissance ${ }^{26} \ldots$ L'architecte Thiollet décrit la maison de son collègue Frœlicher: «Les figures sont du temple de Minerve à Athènes; les griffons, vases, candélabres et autres ornemens sont tirés des monumens de Rome, ainsi que les frises, composées de grands rinceaux d'ornemens; d'autres sont pris d'après des monumens de la renaissance $[s i c] \|^{27}$. Les cahiers d'ornement sont un vecteur essentiel de diffusion. Architectes-ornemanistes, sculpteursornemanistes, les auteurs, Chenavard, Krafft, Romagnési, Normand, Thiollet... copient les motifs byzantins et persans des manuscrits de la Bibliothèque Royale, fréquentent l'atelier de moulage du Musée du Louvre, réalisent des relevés de monuments in situ... Dessinateurs talentueux, ils interprètent, réinventent, affirmant leur volonté d' " élever le goût ». Avec leurs éditeurs, qui font graver ou lithographier des ornements d'artistes européens des siècles passés, ils facilitent l'utilisation des modèles par tous les artistes et tous les métiers: indexation des motifs, dimensions, jusqu'aux cotes d'épaisseur pour les reliefs. Les recueils suggèrent parfois le matériau le mieux adapté ; de fait, les formes pleines de certains motifs semblent appeler la fonte ou la pierre, d'autres plus fins, le carton-pierre ou le fer forgé. Parallèlement, des maisons d'édition spécialisées publient des traités d'architecture, parfois complétés de recueils de menuiserie et de serrurerie ${ }^{28}$. "Plus jolies maisons », «modèles de serrurerie choisis parmi ceux que Paris offre de plus remarquable », l'émulation se crée par l'exemple. Édités dans plusieurs pays, souvent bilingues, ces recueils donnent à voir un «instantané » de la ville, précieux témoins de ses évolutions. Mais qui adapte, traduit en un modèle en trois dimensions l'ornement choisi : les dessinateurs et modeleurs des fabriques, ou bien des artistes connus ne dédaignant pas de compléter leurs revenus? Jean-Baptiste Louis Plantar, dernier sculpteur des bâtiments du Roi, actif sur les grands projets de la monarchie de Juillet, est ainsi mentionné par Thiollet comme auteur de certains modèles du fondeur Ducel. Et plus tard, Pradier, Mathurin Moreau ou Liénard sacrifieront aussi à l'art industriel. Les fondeurs se déterminaient sans doute en fonction de la complexité de la composition et du prix du modèle 29 .

\section{La fonte ornementale est-elle sincère?}

Balzac situe l'action de César Birotteau dans les premières années de la Restauration ; l'ornement de la porte d'entrée est alors un signe de bon goût et de modernité adressé par son personnage au visiteur. La fonte participe aux changements accélérés de la société et revendique une légitimité face aux matériaux nobles de l'art. Mais, si l'architecte Eck admire en la flèche de Rouen

${ }^{26}$ Le fondeur André propose ainsi un choix de "têtes" en médaillons de panneaux : “Marie Stuart, Jean de Bourgogne, Page, Diane de Poitiers, Léonard Vinci [sic], Henri II ».

27 Jean-Charles Krafft, Choix de maisons et d'édifices publics..., $3^{\mathrm{e}}$ volume par François Thiollet, Paris, Bance aîné, 1838, p. 32.

${ }^{28}$ Voir l'ouvrage de François Thiollet : Serrurerie et fonte de fer récemment exécutées, Paris, chez Bance, 1832 ; à noter que fonte et fer forgé étaient souvent réunis sous le terme général de serrurerie.

29 «qui peut quelquefois s'élever jusqu'à 1000 ou 2000 francs avec les frais de dessin, de sculpture, de fonte, de ciselure et d'assemblage [...]. Souvent il arrive qu'un modèle n'est plus de mode, avant qu'on ait vendu assez de pièces pour le payer ", André Guettier, De la fonderie telle qu'elle existe aujourd'hui en France et de ses nombreuses applications à I'industrie, Paris, Carilian-Goeury et Dalmont, 1844, p. 253. 
une «admirable dentelle métallique, ouvrage aussi hardi que grandiose $»^{30}$, Didron la qualifie d'« effroyable flèche en fonte qui écrase cette cathédrale si belle $»^{31}$. Débats et critiques vont s'amplifiant à mesure que la fonte investit la ville jusqu'à imposer son omniprésence à la fin du siècle. Lui reproche-t-on ses lignes moins nettes que le fer forgé, ses formes grêles, son relief moins précis que le bronze, ses surépaisseurs de peinture, la "confusion des matériaux » qu'elle crée? Plus encore, sa reproduction en série, son absence de discernement ${ }^{32}$, sans doute aussi la banalisation des modèles du passé qu'elle met à la portée de tous. On dénonce aux Tuileries les «massifs balcons en fonte de fer qui déparent si ridiculement la façade d'un palais de roi» et le " siècle du postiche " $^{33}$. Nombre des acteurs de l'époque sont nés sous l'Ancien Régime; le procès en sincérité est-il à mettre au compte d'une difficulté à s'abstraire de modèles prestigieux, d'un rejet de la modernité ? Ce n'est pas si simple. L'ingénieur Polonceau lui-même s'étonne que la municipalité veuille ajouter des ornements à son ouvrage : «Les ornements quelconques ajoutés à un pont ne peuvent pas plus le rendre monumental, ni ennoblir son architecture, que des diamants et des fleurs ne peuvent embellir une femme sans beauté, sans tournure, et sans grâce $»^{34}$. Et, au tournant de la deuxième moitié du siècle, le comte de Laborde, ancien directeur du musée des Antiques, estime qu'« au point de vue de l'art, la fonte qui imite le fer forgé se place visà-vis de ce beau métal dans la position qu'occupe le strass à côté du diamant, le papier mâché à côté de la sculpture en pierre, le caoutchouc durci à côté du bois sculpté. La fonte de fer s'est réduite au rôle de contrefaçon. Qu'elle se couvre de peinture ou de dépôts de cuivre, qu'elle imite le fer forgé ou le bambou, peu importe, c'est toujours une contrefaçon, ce n'est pas franc. Or, l'art veut avant tout la franchise, et la fonte de fer et de zinc porte la peine de ses prétentions. Elle n'a pas osé être elle-même, elle a copié les autres, et n'a pas su se faire considérer pour ses avantages propres et ses qualités particulières. ${ }^{35}$ » Pourtant, à Rouen, la flèche affirme la présence de la cathédrale dans l'esprit des bâtisseurs gothiques. Dans le château d'Adélaïde d'Orléans à Randan, elle déploie tout son potentiel structurel et ornemental : candélabres, grilles, jardinières, escaliers, meubles des cuisines, véranda, serres, grands vases du jardin... À Saint-Vincent-de-Paul, elle participe à une synthèse à la fois novatrice et imprégnée des modèles classiques. Alavoine et Hittorff ont su discerner et mettre en valeur le génie propre d'un matériau à la fois résistant et "plastique ${ }^{36}:$ : J'ai de la pierre, j'élève une pyramide en pierre ; vous me donnez du bois, je construis un clocher en bois, qui ne ressemble pas à un clocher en pierre. Enfin, j'ai pour matière unique de la fonte, et je fabrique une flèche suigeneris, qui ne ressemble, ni à une flèche en pierre, ni à une flèche en bois », dit Alavoine à Eustache de La Quérière ${ }^{37}$. Dans les galeries, les théâtres, aux façades des immeubles, elle s'intègre à un paysage urbain cohérent et contribue à fonder une esthétique nouvelle. À la différence peut-être de sa période d'apogée dans la deuxième moitié du siècle, la fonte

${ }^{30}$ C. L. G. Eck, op. cit. note 13, p. 26.

${ }^{31}$ Didron aîné, Annales archéologiques, v. 7, Paris, au Bureau des Annales archéologiques, 1847, p. 119.

${ }^{32}$ Les Observations sur les vases de la Serrurerie de Thiollet décrivent ainsi les modèles illustrés: "Égypte ancienne, anciens Grecs, vases gaulois et romains [...], ornements moresques, règne de Louis XIV, règne de Louis XV, vases du Musée des Antiques, vases Médicis", et même: "vases qui caractérisent le goût du XIX siècle", op. cit. note 28, planches 59 et 60 .

${ }^{33}$ Journal des artistes, 1832, vol. 2, n¹5, p. 265.

34 Antoine-Rémy Polonceau, Notice sur le nouveau système des ponts en fonte..., Paris, Carilian-Goeury, 1839 , p. 86.

${ }^{35}$ Comte de Laborde, Exposition Universelle de 1851, Travaux de la commission française sur I'industrie des nations, tome VIII, XXX Jury, Beaux-Arts, Paris, Imprimerie Impériale, 1856,

p. 834.
${ }^{6}$ Cf. Eck, op. cit. note 13, p. 76 : “ Cet homme ingénieux [le fondeur ChristopheFrançois Calla] est enfin parvenu à pétrir, pour ainsi dire, la fonte avec autant de facilité que la complaisante argile à laquelle il emprunte la création de ses nombreux sujets ».

${ }^{37}$ E. de la Quérière, op. cit. note 11, p. 2. 
ornementale ne nous semble pas à ses débuts devoir être "lue » de manière autonome, mais comme un maillon d'une dynamique architecturale et urbaine. Son histoire est singulière, au cœur d'un débat entre art et industrie qui va s'amplifiant à mesure que le siècle avance. La "sincérité » du matériau, dans cette période de paix retrouvée, n'est-elle pas à rechercher aussi, et surtout, dans sa contribution essentielle à l'assainissement de la ville, à un confort inédit accessible au plus grand nombre, et à de nouveaux décors éclectiques et colorés qui embellissent le quotidien?

\section{Trois corpus parisiens}

\section{Les églises de l'architecte Godde}

Architecte de la ville de Paris sous la Restauration, Étienne-Hippolyte Godde construit plusieurs églises et en rénove d'autres, en rupture avec la relative indifférence de l'Empire pour le patrimoine religieux. Les nouveaux édifices, d'inspiration classique, ont une apparence austère. Les contraintes d'économie favorisent le choix de la fonte pour les clôtures du chœur et des chapelles, et les grilles massives font regretter à Didron le fer forgé et le cuivre : « telle église, qui eût demandé des années pour être meublée et décorée, afin de permettre aux ressources de suffire aux dépenses, est rapidement achevée, grâce à la fonte, aux compositions toutes faites et aux produits de pacotille $»^{38}$. Mais l'architecte innove, en introduisant en contexte religieux un matériau inédit. La polychromie du décor ne nous est pas parvenue dans son intégrité : Thiollet indique que les ornements de Notre-Dame-de-Bonne-Nouvelle et Saint-Pierre-du-Gros-Caillou étaient dorés et les fers peints en bleu ${ }^{39}$. Si les palmes de la première sont toujours dorées, les grilles de la seconde sont uniformément noires. Seule SainteÉlisabeth restitue l'esprit d'origine : le motif de la croix centrale inscrite dans un médaillon d'où rayonnent quatre palmes et quatre épis, répété à quelques variations près dans les édifices du corpus, est coloré en rouge, vert et or (fig. 6). Fait notable, Sainte-Élisabeth est aussi la première église où cohabitaient fonte d'ornement et lave émaillée : Hittorff s'en est-il inspiré pour son chef d'œuvre Saint-Vincent-de-Paul ?

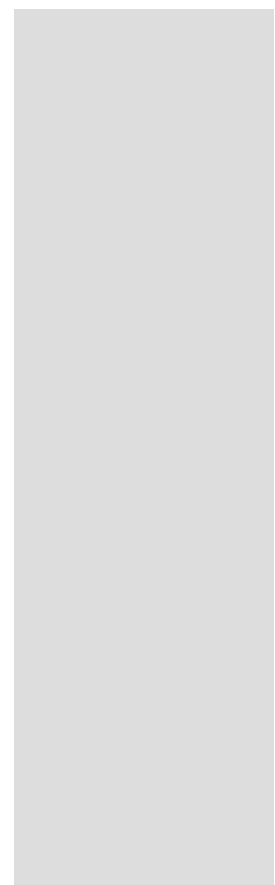




\section{Le cimetière du Père Lachaise}

Au cimetière du Père Lachaise, la fonte ornementale est omniprésente. À défaut de datation directe, quelques ouvrages contemporains 40 permettent d'identifier le patrimoine d'époque Restauration et monarchie de Juillet ${ }^{41}$, et de mettre en perspective l'évolution du décor avec celui de l'architecture civile. La porte du monument de L'Espine, ornée de sabliers ailés et de figures de pleureuses, est un bel exemple précoce de décor funéraire en fer et fonte (fig. 7). Des compositions singulières comme les ancres et rames entrecroisées du monument Rosily cohabitent avec la symbolique funéraire classique des flambeaux, vases, chauve-souris, lampes à huile des sépultures Bassano, Lanjuinais ou Morainville. Les monuments des métallurgistes Ardaillon et Chagot sont entièrement construits en fonte, ce dernier signé, fait exceptionnel ${ }^{42}$. D'abord limitée à quelques réalisations au dessin original, la fonte devient en quelques années le matériau incontournable du décor funéraire. Au hasard des allées, le promeneur peut mesurer l'évolution, de la simplicité des premiers monuments jusqu'à la complexification ultérieure des décors, et aussi l'effacement progressif de l'œuvre artisanale unique devant les compositions de série. 


\section{Les façades des immeubles Restauration et Louis-Philippe}

La fonte ornementale investit au même moment les façades des immeubles bourgeois de la capitale. Nouveaux quartiers, nouvelles rues lui ouvrent de larges territoires: balcons, appuis, panneaux de porte, impostes... Rapidité et économie de construction sont essentielles pour les immeubles de rapport: la fonte «sur catalogue » s'avère un matériau idéal. Ce patrimoine modeste est si familier qu'on n'y prête guère attention; notre visite nous conduit dans les quartiers de la Nouvelle-Athènes, Saint-Georges, Poissonnière, boulevard Beaumarchais, rue Rambuteau, rue du Pont Louis-Philippe. Nous pouvons constater que de nombreux balcons et panneaux figurent aux catalogues d'André (fig. 8), de Savanne et de Ducel. Toujours peinte, parfois dorée, la fonte participe à un nouveau vocabulaire décoratif, répondant aux motifs sculptés dans le bois des portes, la pierre ou le stuc des linteaux. Rue des Francs-Bourgeois, les vases à guirlandes de l'hôtel de Sandreville ou les dragons affrontés de l'hôtel d'Almeras semblent avoir influencé, à quelques décennies d'intervalle, les fontes ornementales de la rue Rambuteau voisine. Le motif de la tête Renaissance en fort relief inscrite dans un médaillon est un succès des premiers catalogues; des compositions en panneaux symétriques : portrait d'homme accompagné de son pendant féminin, peuvent ainsi s'observer boulevard Beaumarchais. Lotie dans les premières années de la monarchie de Juillet, la rue du Pont Louis-Philippe donne à voir de belles fontes à jour d'André, Savanne et Ducel: oiseaux exotiques affrontés picorant des fruits, putti adossés nourrissant des animaux... La comparaison des ouvrages permet d'observer la structure modulaire des compositions: symétrie autour d'un axe vertical, registres superposés, et le foisonnement apparent d'un décor assemblé à partir d'un nombre limité d'éléments de base. Si beaucoup de ces panneaux sont parvenus jusqu'à nous, les surépaisseurs d'une peinture souvent uniformément noire ou grise dénaturent la précision du moulage et le décor voulu par l'architecte.

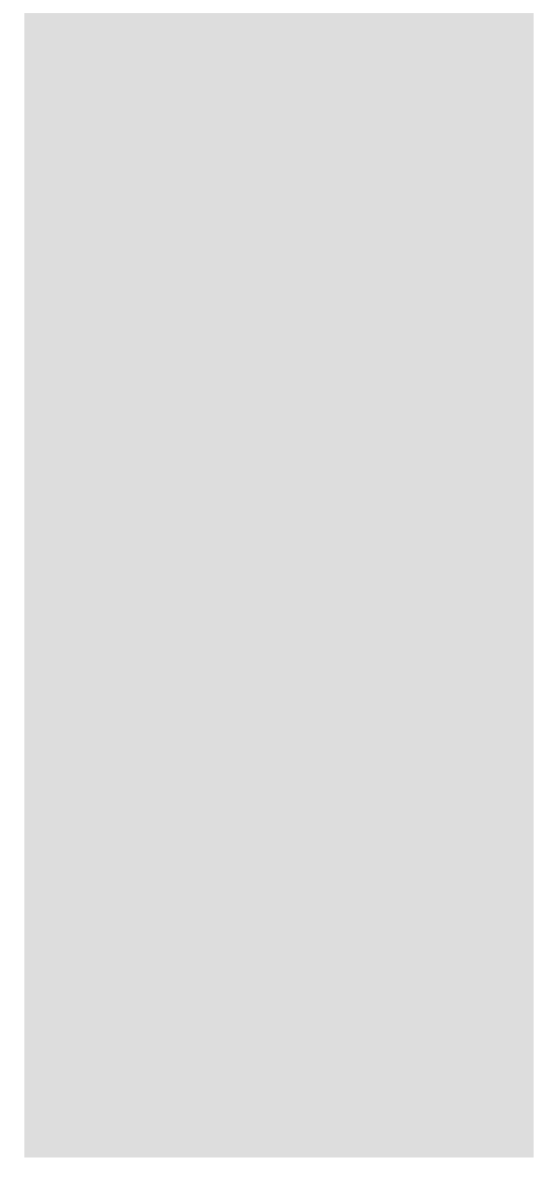




\section{Pour citer cet article}

\section{Référence électronique}

Jean-Paul Zitt, «Les débuts de la fonte ornementale en France, de l'Empire à la monarchie de Juillet », Cahiers de l'École du Louvre. Recherches en histoire de l'art, histoire des civilisations, archéologie, anthropologie et muséologie [en ligne] no 1 , septembre 2012, mis en ligne le 15 septembre 2012

http://www.ecoledulouve.fr/cahiers-de-l-ecole-du-louvre/numero1 septembre2012/Zitt.pdf

\section{(c) creative \\ (B.) $\ominus$ (오}

\section{L'auteur}

Après une carrière professionnelle dans l'industrie, Jean-Paul Zitt a rejoint l'École du Louvre, avec pour spécialité de premier cycle le patrimoine technique et industriel. Intéressé par le débat art et industrie et ses acteurs, par l'ornement et la diffusion des modèles, il a choisi en master un thème de recherche croisant ces différentes problématiques : la fonte de fer ornementale, à un moment singulier : le début de la révolution industrielle en France. 